\title{
Giant Magneto Impedance Effect of Co-Based Metallic Fiber Under Bias Magnetic Field
}

\author{
Shuling Zhang ${ }^{a, b *} \oplus^{\infty}$, Weiye Chen ${ }^{c}$, Mingkun Qiu ${ }^{b}$ \\ ${ }^{a}$ School of Mechanical \& Automotive Engineering, Qingdao University of Technology, Qingdao, 266520, China \\ ${ }^{b}$ School of Mechanical Engineering, Ningxia University, Yinchuan, 750021, China \\ ${ }^{c}$ School of Material of Science and Engineering, North Minzu University, Yinchuan, 750021, China
}

Received: November 20, 2018; Revised: May 10, 2019; Accepted: May 19, 2019

\begin{abstract}
The giant magneto impedance, GMI, effect of Co-based metallic fibers prepared by melt-extracted technology is investigated under different bias direct electrical current $\mathrm{I}_{\mathrm{b}}$. The GMI curves have single peaks which are symmetric about zero field at and below $1 \mathrm{MHz}$. However, the bimodal GMI curves which have asymmetric peaks are observed when $I_{b}$ is over $2 \mathrm{~mA}$ at $0.1 \mathrm{MHz}$. And asymmetric GMI, AGMI, effect is also appeared at $1 \mathrm{MHz}$ when $\mathrm{I}_{\mathrm{b}}$ just is $1 \mathrm{~mA}$ and the field sensitivity of GMI effect is increased 5.6 times. The GMI effect and magnetization process is affected by the bias magnetic field profoundly induced by $\mathrm{I}_{\mathrm{b}}$, which is correlated with the outer shell domain structures with circumferentially magnetization easy axis existed in the outer shell of Co-rich magnetic wires with low and negative magnetostriction. Below $1 \mathrm{MHz}$, only if the bias current is over $1.5 \mathrm{~mA}$ which induces a magnetic circumferential magnetic field is above the coercivity of $14.4 \mathrm{~A} / \mathrm{m}$, the circumferential magnetization and GMI effect can experience the bias magnetic field effect. Above $1 \mathrm{MHz}$, the skin effect gets stronger and significantly decreases the total participants of circular magnetization process in the fibers and consequently, the circular outer shell magnetization process gets much sensitive to circular bias magnetic field. AGMI effect with high field sensitivity is realized due to the bias current.
\end{abstract}

Keywords: GMI effect, magnetization process, field sensitivity, metallic fibe

\section{Introduction}

The large change in the impedance of soft magnetic conductors has been referred to as the giant magneto impedance, GMI. When a magnetic conductor with a highfrequency alternating current is subjected to an external magnetic field, the impedance of the material may change ${ }^{1}$. Since the discovery of the GMI effect in Co-based magnetic metal fibers, numerous studies have been inspired on the magnetization process of metal fibers to investigate its origin and determinants ${ }^{1,2}$. In the case of a Co-based magnetic fibers with a negative magnetostriction constant, it is assumed that there is an inner core magnetized along the wire axis and an outer shell where magnetization points to in the circumferential direction ${ }^{3,4}$. The magnetization process of these metal fibers is studied theoretically and experimentally and the possible magnetic domain structure is proposed and then observed ${ }^{4-8}$. The unique magnetization process of this kind of fibers makes it has important application in the development of magnetic sensors. However, Zhukova et al recently have found that the magnetization process of this kind of fibers is indeed complex and not fully understood and mastered $^{9}{ }^{10}$. They found that due to the interplay between the shape axial and circumferential anisotropies, amorphous ferromagnetic fibers exhibit a rich variety of domainline magnetic phases ${ }^{11}$. And the changes induced in the outer domain shell can impact on the magnetization in the inner core.
An observed domain wall propagation induced by the current flowing through the metallic fibers is explained considering the influence of an Oersted magnetic field on the outer domains ${ }^{10}$. Therefore, it is necessary to study and explore the magnetization process of metal fibers. All scientific research eventually serves humans. The concept of GMI effect sensor has been proposed long ago, but mass production and application have been rarely reported. In particular, highperformance GMI sensors are reported theoretically and are not widely used in industry and life. Based on the study of GMI effect, this paper will investigate the magnetization process of metal fiber, explore the influence of magnetization process on electromagnetic effect, reveal possible ways to improve GMI effect, and provide reference and support for the rapid development of GMI sensors.

\section{Experimental Detail}

Impedance analysis is performed using a HP4192 impedance analyzer at room temperature. The test sample is $25 \mathrm{~mm}$ in length, 32 microns in diameter, and the applied external magnetic field is provided by Helmholtz coil. The maximum magnetic field is $320 \mathrm{~A} / \mathrm{m}$, the driving current is from $1 \mathrm{~mA}$ to $10 \mathrm{~mA}$, the bias direct current is from $1 \mathrm{~mA}$ to $10 \mathrm{~mA}$, and the frequency is from $0.1 \mathrm{MHz}$ to $10 \mathrm{MHz}$. In order to prevent the influence of environment field on the testing process, the fiber is placed axially perpendicular to the geomagnetic field. 
The GMI ratio is defined as:

$$
\Delta Z / Z=\frac{Z\left(H_{e x}\right)-Z\left(H_{-\max }\right)}{Z\left(H_{-\max }\right)} \times 100 \%
$$

Where $Z\left(H_{e x}\right)$ and $Z\left(H_{\text {-max }}\right)$ are impedances at external field of $H_{e x}$ and the maximum negative field. And the field sensitivity is defined as:

$$
S=\frac{d Z}{d H_{e x}} \cdot i_{a c}
$$

Where $i_{a c}$ is the current amplitude.

\section{Results and Discussion}

Figure 1 shows the GMI effect at different frequencies. As the frequency increases, the magnetic impedance gradually increases under the same external magnetic field. At $0.1 \mathrm{MHz}-$ $1 \mathrm{MHz}$, GMI curves gradually decrease with the increase of magnetic field intensity, and the maximum impedance value is obtained at zero magnetic field, showing a single peak GMI curve. When the frequency is $5 \mathrm{MHz}$, the magnetic impedance increases with the increase of the external magnetic field and gradually decreases after reaching the maximum value. There are two impedance peaks at different magnetic fields. Therefore, two peaks, namely the bimodal structure GMI effect, can be observed.

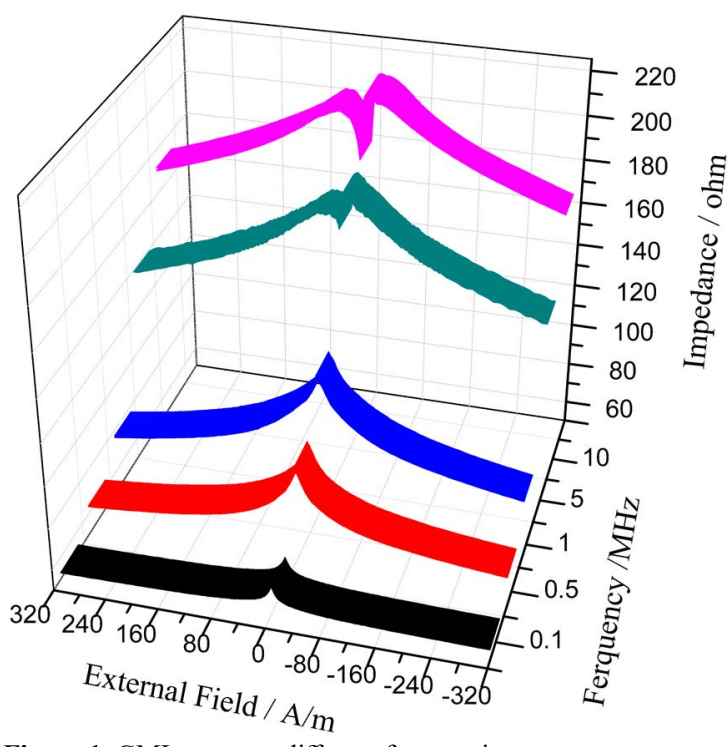

Figure 1. GMI curves at different frequencies

The GMI effect comes from the change of circular permeability with external magnetic field, so it is closely related to the magnetization process ${ }^{12,13}$. There is a "coreshell" magnetic domain structure for these kind of the Cobased micro fibers ${ }^{4}$. The core is magnetized longitudinally, and the shell has an annular easy magnetization direction.
The magnetization process is different with frequency. Under the axial magnetic field, axial magnetization of the core domains is easy and the circular magnetization will be greatly affected by the intensity of the magnetic field. At lower frequencies $(<1 \mathrm{MHz})$, when the axial magnetic field is zero, the circular magnetization reaches its maximum. Therefore, the impedance reaches its peak at zero magnetic field showing single-peak GMI effect. As the frequency increases, the skin effect gradually becomes obvious, and the influence from circumferential magnetic field generated by the driving current on the magnetization process is strengthened, consequently. And the circumferential magnetization process in the shell is improved. However, it is necessary to pay attention to the magnetic interaction between the shell and the core magnetization at the same time which will also induces demagnetization ${ }^{9,14,15}$. Therefore, circumferential magnetization can continue only if the applied circular magnetic field reaches a threshold value, that is, the magnetization process will produce a huge change, at which the impedance reaches a peak value. Then there is bimodal structure GMI curve when the frequency reaches $\mathrm{MHz}$, as shown in Fig.1 (b).

As aforementioned, the enhancement of circumferential magnetization process can theoretically affect the GMI response, as shown in Figure 1(b) and Figure 2. With the increase of the driving current, both the impedance values and GMI curves are altered. When there is a bias current of $2 \mathrm{~mA}$ or the driving current increases to $2 \mathrm{~mA}$, the GMI effect is significantly increased and the curves become asymmetry. More obviously, as the bias current increases from $1 \mathrm{~mA}$ to $10 \mathrm{~mA}$, the GMI curves gradually change from single peak to double peaks. At $1 \mathrm{MHz}$, a bias current of $1 \mathrm{~mA}$ can change GMI curve from single peak to asymmetric double peaks. Meanwhile, as the bias current increases to $2 \mathrm{~mA}$, the sensitivity increases gradually from $0.6 \times 10^{-3} \mathrm{~V} /(\mathrm{A} / \mathrm{m})$ to $3.6 \times 10^{-3} \mathrm{v} /(\mathrm{A} / \mathrm{m})$.

The GMI curve of $0.1 \mathrm{MHz}$ shows that the impedance value at the negative magnetic field is lower than those at the positive parts, that is, the impedance value does not have strict symmetry near the zero magnetic field, which indicates that the circular domain of the shell of Co-based fiber has spiral anisotropy ${ }^{16}$. The circumferential magnetization process of domains with magnetization angle less than $90^{\circ}$ between surface domains and the bias current induced magnetic field in the shell is enhanced but the circumferential magnetization process is decreased in the shell domains which easy direction of magnetization deviates greatly from the direction of the constant external magnetic field. Circumferential magnetization process of special parts is strengthened which induces and increases the asymmetric magnetization process at negative and positive the external fields, as shown in Fig. 3. The asymmetrical characteristic of GMI curve is elongated and AGMI effect gradually increases. 

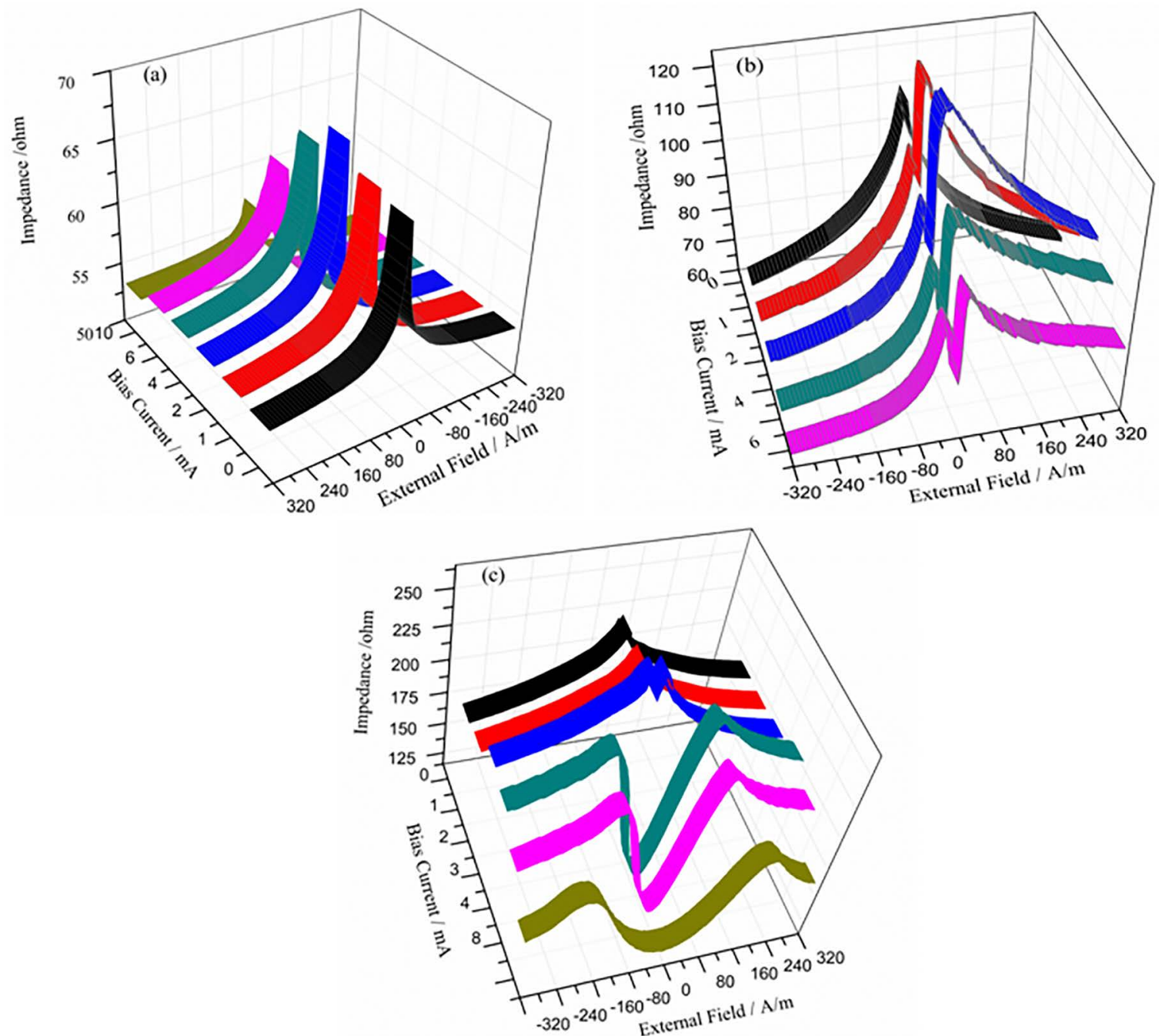

Figure 2. GMI curves under different bias currents: (a)0.1 MHz, (b) $1 \mathrm{MHz},(\mathrm{c}) 5 \mathrm{MHz}$

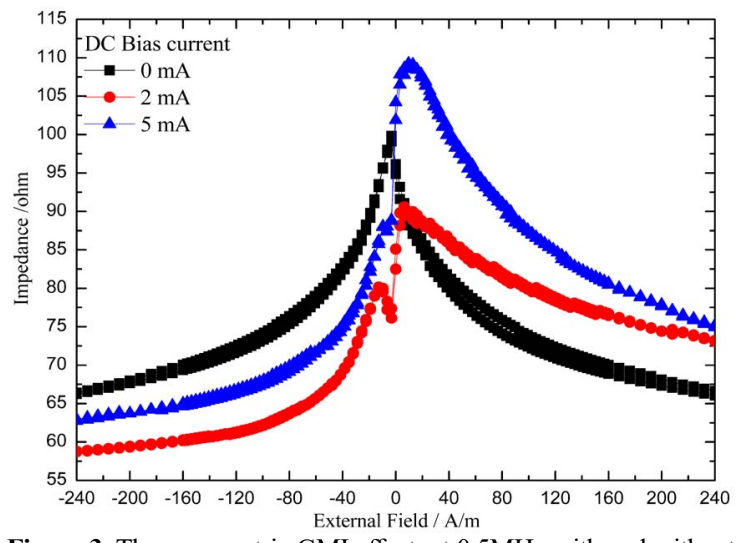

Figure 3. The asymmetric GMI effect at $0.5 \mathrm{MHz}$ with and without bias current

When the bias current is $2 \mathrm{~mA}$, the GMI effect and its asymmetry feature near the zero magnetic field are more pronounced and stronger. However, when the circumferential field created by the current is too more powerful, domain wall motion in the shell finally is suppressed together with the asymmetry, resulting in a reduction of the permeability and thus of the GMI effect with the increase of driving or bias current ${ }^{9,17}$, as shown in Fig. 4.

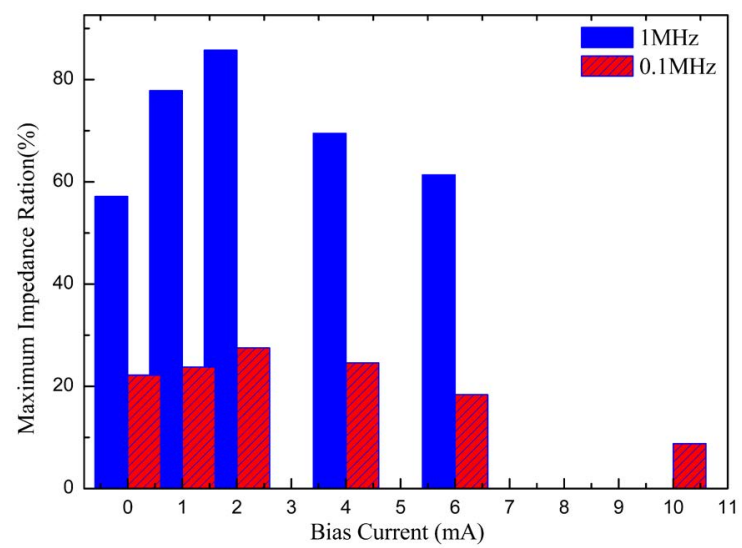

Figure 4. The maximum GMI ratio under different bias current 
Only if the induced circumferential magnetic field by bias current is above samples coercivity and therefore can affect the magnetization of the outer domains in shell with circumferential magnetization direction ${ }^{14,18}$. The coercivity of such metal fibers is $14.4 \mathrm{~A} / \mathrm{m}$ measured by experiments. Therefore, when the bias current is greater than $1.5 \mathrm{~mA}$, the induced circumferential magnetic field is equal to the coercivity. Although the bias current of $1 \mathrm{~mA}$ can influence the circular magnetization process of fibers, the effect on GMI is not enough to change its main feature. When the bias current is greater than $1.5 \mathrm{~mA}$, the induced magnetic field is greater than the coercivity, which has a huge impact on the circular magnetization process of these Co-based fibers9. As the frequency increases, the skin effect gets strong, so even a small current can produce enough circular magnetic field to affect the circumferential magnetization process and GMI effect.

It is found that the control of the domain wall motion induced by a magnetic field directly into the magnetic element is a recent issue in magnetism as well as a technological challenge $^{11}$. In order to investigate the GMI effect and surface domain magnetization, the influence of driving alternating amplitude on GMI effect is analyzed, as shown in Fig. 5.

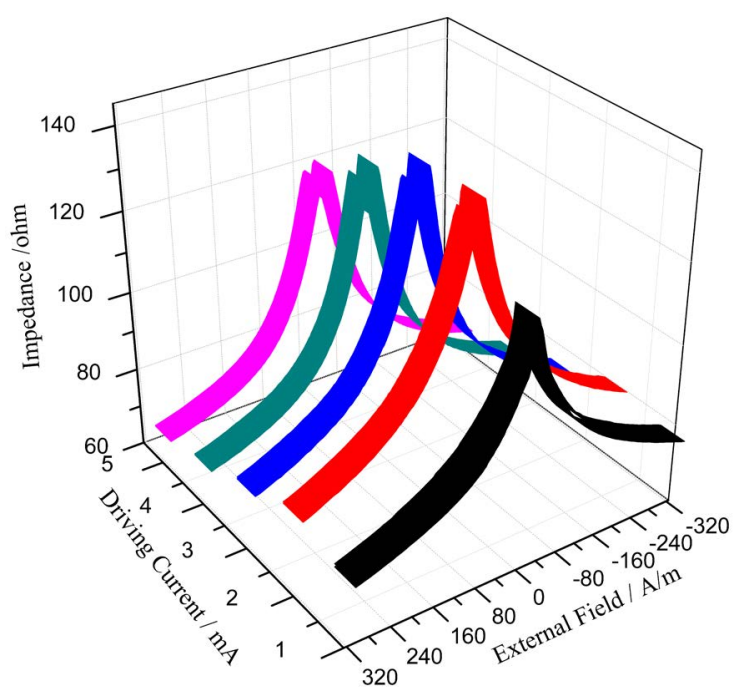

Figure 5. GMI effect at different driving currents $(1 \mathrm{MHz})$

For the GMI effect of ac excitation, the alternating current itself generates an alternating circumferential magnetic field on the surface of the fiber. Therefore, the excitation current amplitude increases, and the surface circumferential magnetic field intensity increases, which should have a similar effect to the bias current. Experimental studies show that AGMI effect can be enhanced by increasing the excitation current amplitude, and GMI curve changes from single peak to double peaks, which further indicates that the circumferential magnetization process of shell domains is an important factor influencing the GMI effect of this kind of Co-based fibers.
It is worth noting that the effect of ac current amplitude is not as significant as that of bias current, and the effect of bias current on GMI effect is much more significant. Excitation current is an alternating current with frequency, which density is affected by frequency due to the skin effect, but the bias current is a direct current, so the direction is constant. Therefore, the circumferential magnetic field generated by bias current has a stronger influence on the circular domain of shell, and the AGMI effect obtained is also more obvious. This analysis further indicates that the high sensitive GMI effects can be effectively promoted by controlling surface domains and its circular magnetization process.

\section{Conclusions}

Based on the analysis of the influence of circular magnetic field induced by bias and driving currents on GMI effect, it is found that the shell domains of Co-rich fibers are a kind of surface domains with spiral anisotropy, and the GMI curve is single peak at low frequency and double peaks at high frequency. The circumferential magnetic field generated by the current influences the circumferential magnetization process of the shell domains, so that part of the magnetization process is strengthened and GMI effect is enhanced along with the appearance of asymmetry. However, the GMI effect is much more significantly affected by the uniform and constant bias current density. The results show that the control of circumferential magnetization process of the surface domains is the key to improve GMI effect and field sensitivity of Co-rich fibers.

\section{Acknowledgements}

The authors would like to express their appreciation to Prof. Yelon and Menard from University of Montreal of Canada for support and assistance in impedance experiment. This work was financially supported by the National Natural Science Foundation of China (Nos. 51861031, 51604159) and the Natural Science Foundation of Ningxia (No: 2018AAC03056)

\section{References}

1. Beach RS, Berkowitz AE. Giant magnetic field dependent impedance of amorphous $\mathrm{FeCoSiB}$ wire. Applied Physics Letters. 1994;64(26):3652-3654.

2. Kurlyandskaya GV. Giant magnetoimpedance for biosensing: Advantages and shortcomings. J Journal of Magnetism and Magnetic Materials. 2009;321(7):659-662.

3. Chizhik A, Gonzalez J, Yamasaki J, Zhukov A, Blanco JM. Vortex-type domain structure in Co-rich amorphous wires. Journal of Applied Physics. 2004;95(5):2933-2935.

4. Chizhik A, Garcia C, Gonzalez J, Zhukov A, Blanco JM. Study of surface magnetic properties in Co-rich amorphous microwires. Journal of Magnetism and Magnetic Materials. 2006;300(1):e93-e97. 
5. Gómez-Polo C, Vázquez M, Chen DX. Directionally Alternating Domain Wall Propagation in Bistable Amorphous Wires. Applied Physics Letters. 1993;62(1):108-109.

6. Chizhik A, Gonzalez J. Modeling of magnetization reversal in amorphous wires. Physica B: Condensed Matter. 2006;372(1-2):341-344.

7. Zhukova V, Blanco JM, Rodionova V, Ipatov M, Zhukov A. Domain wall propagation in micrometric wires: Limits of single domain wall regime. Journal of Applied Physics. 2012;111(7):07E311.

8. Chizhik A, Zablotskii V, Stupakiewicz A, Dejneka A, Polyakova $\mathrm{T}$, Tekielak M, et al. Circular domains nucleation in magnetic microwires. Applied Physics Letters. 2013;102(20):202406.

9. Zhukova V, Blanco JM, Chizhik A, Ipatov M, Zhukov A. Current induced domain wall propagation in Co-rich amorphous microwires. AIP Advances. 2017;7(5):056026.

10. Zhukova V, Blanco JM, Chizhik A, Ipatov M, Zhukov A. AC-current-induced magnetization switching in amorphous microwires. Frontiers of Physics. 2018;13:137501.

11. Chizhik A, Zablotskii V, StupakiewiczA, Gómez-Polo C, Maziewski A, Zhukov A, et al. Magnetization switching in ferromagnetic microwires. Physical Review B. 2010;82(21):212401.

12. Zhukov A, Talaat A, Churyukanova M, Kaloshkin S, Semenkova $\mathrm{V}$, Ipatov $\mathrm{M}$, et al. Engineering of magnetic properties and
GMI effect in Co-rich amorphous microwires. Journal of Alloys and Compounds. 2016;664:235-241.

13. Chizhik A, Zhukova V, Zhukov A, Gonzalez J, Gawroński P, Kułakowski K, et al. Surface magnetic structures induced by mechanical stresses in Co-rich microwires. Journal of Alloys and Compounds. 2018;735:1449-1453.

14. Zhukov A, Chizhik A, Ipatov M, Talaat A, Blanco JM, Stupakiewicz A, et al. Giant magnetoimpedance effect and domain wall dynamics in Co-rich amorphous microwires. Journal of Applied Physics. 2015;117(4):043904.

15. Chizhik A, Zhukov A, Gonzalez J, Stupakiewicz A. Control of the domain wall motion in cylindrical magnetic wires. Applied Physics Letters. 2016;109(5):052405.

16. Panina LV. Asymmetrical giant magneto-impedance (AGMI) in amorphous wires. Journal of Magnetism and Magnetic Materials. 2002;249(1-2):278-287.

17. Zhukova V, Ipatov M, Talaat A, Blanco JM, Churyukanova M, Taskaev S, et al. Effect of stress-induced anisotropy on high frequency magnetoimpedance effect of $\mathrm{Fe}$ and Co-rich glass-coated microwires. Journal of Alloys and Compounds. 2018;735:1818-1825

18. Chizhik A, Zhukov A, Gonzalez J, Stupakiewicz A. Control of reversible magnetization switching by pulsed circular magnetic field in glass-coated amorphous microwires. Applied Physics Letters. 2018;112(7):072407. 